\title{
BENIGN TUMOR OF NOSE AND PARANASAL SINUS IN A CHILD: A CASE REPORT
}

\author{
Mujtaba Khan ${ }^{1}$, S. Muneeruddin Ahmed ${ }^{2}$, A. Sesha Prasad ${ }^{3}$, M. Mahendra Kumar, G. Shahul Hameed ${ }^{5}$
}

\section{HOW TO CITE THIS ARTICLE:}

Mujtaba Khan, S. Muneeruddin Ahmed, A. Sesha Prasad, M. Mahendra Kumar, G. Shahul Hameed. "Benign Tumor of nose and Paranasal Sinus in a child: A case report". Journal of Evolution of Medical and Dental Sciences 2014; Vol. 3, Issue 30, July 28; Page: 8403-8407, DOI: 10.14260/jemds/2014/3060

ABSTRACT: Olfactory neuro-blastoma is a rare malignant neoplasm of the neuro-epithelial origin. A 6 year old female presented with a swelling of right cheek with protrusion of right eyeball over a period of one month. Computed Tomography of the Para-nasal sinuses showed expansion of the right maxillary sinus with intra-orbital extension. A diagnosis of olfactory neuroblastoma was established by histopathology and confirmed by Immunohistochemistry. It was Stage C of Kadish (modified) classification. A right medial Maxillectomy was performed and the entire Tumor was removed.

KEYWORDS: Olfactory, Neuro, blastoma, extra nasal invasion, maxillary sinus, Immunohistochemistry, medial maxillectomy.

INTRODUCTION: Olfactory neuro-blastoma is a rare malignant neoplasm of olfactory neuroepithelial origin, accounting for less than $5 \%$ of all the Sino-nasal malignancies. The neoplasm has a bimodal age distribution peaking at age 20 and 50 years respectively. It usually presents with nasal obstruction, recurrent epistaxis and hyposmia. Invasion of the Dura and the anterior cranial fossa is seen in $25 \%$ of the cases. The present report is a rare extra nasal presentation of the tumor.

CASE REPORT: A 6 year old female child presented to the ENT OPD of Govt. E.N.T Hospital Koti, Hyderabad; India with the presenting complaints of swelling of the right cheek since one month and protrusion of the right eyeball since 20 days. There was no history of any numbness over the swelling, no history of loss of vision or diplopia. No history of headache, fever or vomiting could be elicited.

Examination revealed a diffuse swelling over the right cheek measuring about $6 \mathrm{x} 6 \mathrm{~cm}$ extending vertically $1 \mathrm{~cm}$ below the right lower eyelid to $1.5 \mathrm{~cm}$ above the right upper lip and horizontally from the right Naso-labial fold to $8 \mathrm{~cm}$ towards the angle of mandible with obliteration of the right Naso-labial fold. There was no evidence of cranial nerve palsies and palpable cervical lymph nodes. Tests for olfaction were performed and the sense of smell was found to be decreased on the right side.

A provisional diagnosis of an Ossifying Fibroma was made. Computed Tomography scan of the Para-nasal sinuses was performed, which showed evidence of large well enhancing soft tissue density lesion in the right maxilla with thin bony septations measuring 37 x 34 x $33 \mathrm{~mm}$. Irregular destruction of the floor of the orbit and the Antero-right lateral wall of the maxillary sinus. Intraorbital extension of the soft tissue was noted on the right side.

Fine Needle Aspiration (FNAC) was suggestive of a tumor of poorly differentiated origin. Histopathological examination was done, taking the specimen by sub-labial approach. It was suggestive of a small round cell tumor. Immunohistochemistry with Neuron Specific Enolase (NSE) was done to confirm the diagnosis and the tumor showed cytoplasmic positivity in all the areas of the 
tissue. This was highly suggestive of Olfactory Neuroblastoma. A diagnosis of Olfactory neuroblastoma, Kadish (modified) Stage $\mathrm{C}$ was made.

The patient was taken up for excision of the tumor and a medial Maxillectomy was performed using a lateral Rhinotomy approach. The final histopathological examination also confirmed the tumor as olfactory neuroblastoma.

Fig. 1: Pre-operative photograph of the child showing right cheek swelling and protrusion of the right eye.

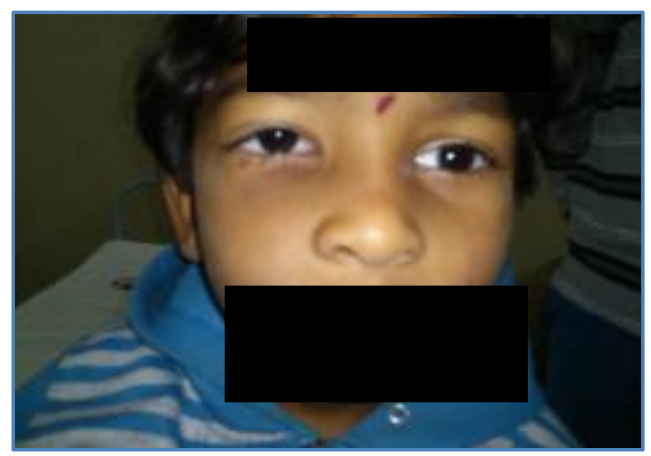

Fig. 1

DISCUSSION: Olfactory Neuro- blastoma, was first described by Berger and Richard in $1924 .{ }^{1}$ Olfactory Neuroblastoma represents less than $5 \%$ of the all Sino-nasal Malignancies. ${ }^{2}$ It arises from the olfactory Neuro-epithelium which extends from the roof of the nose to area of the superior turbinate. Most of the cases described in the literature are of adults.

It has a bimodal age distribution peaking at 20 and 50 years respectively. Unlike most of the Sino-nasal malignancies, it is more common in females. The most common symptoms of the Tumor are nasal obstruction, recurrent epistaxis and headache. ${ }^{3}$ Also called as an Esthesio- neuroblastoma or a neuroendocrine Tumor; it is capable of causing Para-neoplastic.

Fig. 2: CT scan of Para-nasal sinuses showing expansion of the right maxillary sinus with soft tissue attenuation and right Intra-orbital extension.

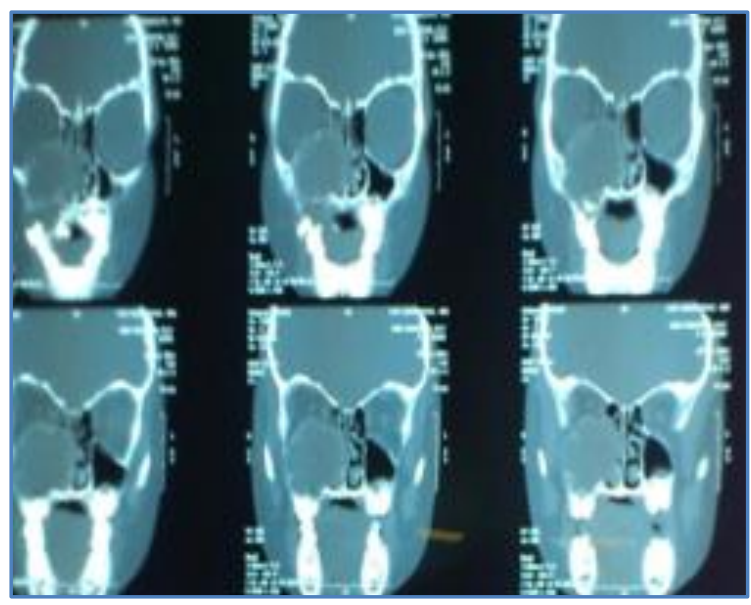

Fig. 2 
SYNDROMES: antidiuretic hormone secretion and hypertension have also been reported. In about $5 \%$ of the patients metastasis to the cervical nodes is evident at the time of the presentation. The diagnosis is predicted by imaging characteristics and the histopathological examination.

High resolution CT and MRI are useful to delineate the exact extent of the Tumor and to define the involvement of the cribriform plate and the anterior cranial fossa. In $25 \%$ of the patients the Dura and the anterior cranial fossa is invaded.

Histopathological examination shows well-circumscribed cell nests and lobules separated by fibro vascular stroma. The lobules may coalesce and interconnect, forming sheets of cells with a prominent capillary network. Rosettes are also typical. Homer Wright rosettes, also called pseudorosettes, are annular arrays of cells surrounding central zones of fibrils.

The neoplastic cells are small or medium size and have pale eosinophilic cytoplasm with chromatin and absent or inconspicuous nucleoli. Most cases show absent or only mild to moderate nuclear pleomorphism and a low mitotic rate. Necrosis is uncommon and is generally seen in poorly differentiated Tumors with high mitotic counts.

Immunohistochemical studies have shown that the cells of olfactory neuroblastoma express neuron-specific Enolase, CD56, synaptophysin and chromogranin. Hyams et al $^{4}$ developed a four point histological grading system based on features such as degree of differentiation, Tumor architecture, mitotic index, nuclear pleomorphism, fibrillary nature of the matrix and Tumor necrosis.

The pathological grade of the Tumor was found to be the most significant prognostic factor, with an 80\% 5-year survival rate and a 40\% 5-year survival rate for low-grade and high-grade Tumors, respectively.

Fig. 3: Histopathological examination showing small monotonous cells with large hyperchromatic nuclei suggestive of small round cell Tumor.

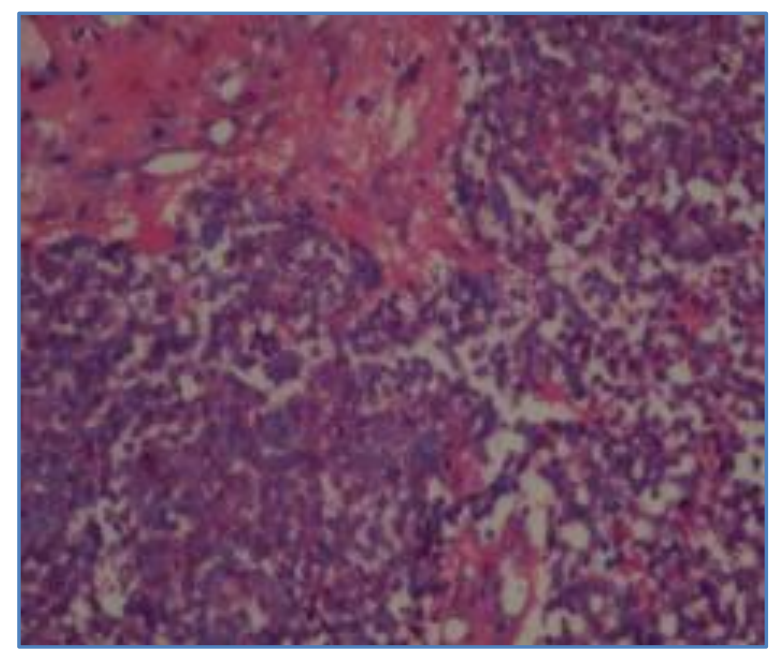

Fig. 3 


\section{CASE REPORT}

Fig. 4: A right medial Maxillectomy was performed using lateral Rhinotomy approach _ Tumor was removed.

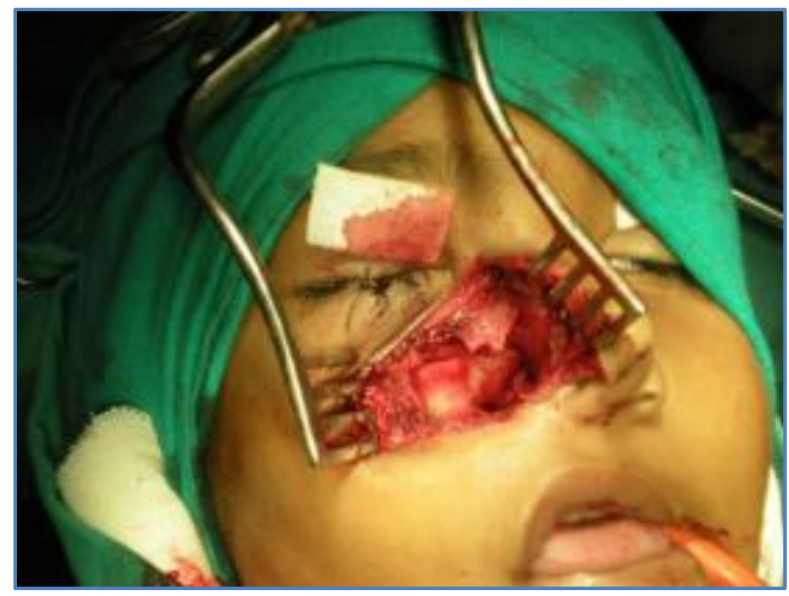

\section{Fig. 4}

Various staging systems have been devised for olfactory neuroblastoma like the Biller et al, Kadish et al and Dulgerov and Calcaterra staging systems. The system proposed by Kadish is simple and is the most commonly used.

\section{Kadish Staging5:}

Kadish A-

Limited to nasal cavity.

Kadish BInvolving nasal cavity and sinuses.

Kadish C- $\quad$ Extension beyond the nasal and Para-nasal sinuses.

Kadish D- $\quad$ Tumor with metastasis to cervical nodes or distant sites.

Regardless of the grade or stage -- with the possible exception of distant metastasis - surgery is the recommended mainstay of therapy in patients harboring these tumors. Surgical treatment alone is effective in cases of low-grade Tumor if Tumor-free margins can be obtained.

The use of adjuvant radiotherapy is supported for low-grade tumors when the margins are close, for residual or recurrent disease, and for all high-grade tumors. Some authors have argued that radiation is best administered preoperatively, whereas others prefer to administer it postoperatively, and yet others recommend postoperative radiotherapy for Stage A tumors and preoperative radiotherapy for higher-stage lesions (Stages B and C).

There have been reports of cases with unresectable lesions in which long-term survival was achieved following a combination of chemo- and radiotherapy. ${ }^{6}$ It appears that high-grade tumors show the greatest response to cisplatin-based chemotherapeutic treatment. ${ }^{7}$

CONCLUSION: Olfactory neuroblastoma is a rare Tumor, presenting with nasal obstruction and epistaxis. In this patient the Tumor presented with a cheek swelling and protrusion of the eye. The possibility of a malignant Tumor of the nose or the Para-nasal sinuses should be sought especially if there is a short history. Surgery followed by radiotherapy is the mainstay of the treatment. 


\section{REFERENCES:}

1. Berger L, Luc R. L' esthesioneuropetheliome olfacif. Bull Cancer 1924; 13: 410 \pm 421 .

2. Bradley PJ, Jones NS, Robertson I. Diagnosis and management of esthesioneuroblastoma. Curr Opin Otolaryngol. Head Neck. Surg. 2003 Apr; 11 (2): 112- 8.

3. Levine PA, Gallagher R, Cantrell RW. Esthesioneuroblastoma: reflections of a 21- year experience. Laryngoscope 109: 1539-1543, 1999.

4. Hyams VJ, Batsakis JG, Michaels L. Tumors of the upper respiratory tract and ear. In: Atlas of Tumor Pathology, Armed Forces Institute of Pathology, 1988.

5. Morita A, Ebersold MJ, Olsen KD, et al. Esthesioneuroblastoma: prognosis and management. Neurosurgery 1993; 32: 706.

6. Zappia JJ, Carroll WR, Wolf GT, et al. Olfactory neuroblastoma: the results of modern treatment approaches at the University of Michigan. Head Neck 15:190-196, 1993

7. McElroy EA Jr, Buckner JC, Lewis JE. Chemotherapy for advanced esthesioneuroblastoma: the Mayo Clinic experience. Neurosurgery 42: 1023-1028, 1998.

\section{AUTHORS:}

1. Mujtaba Khan

2. S. Muneeruddin Ahmed

3. A. Sesha Prasad

4. M. Mahendra Kumar

5. G. Shahul Hameed

\section{PARTICULARS OF CONTRIBUTORS:}

1. Senior Resident, Department of ENT, Osmania Medical College, Hyderabad and Now Consultant in ENT, Hyderabad.

2. Professor and HOD, Department of ENT, Viswabharathi Medical College, Kurnool.

3. Associate Professor and I/C Professor, Department of ENT, Kurnool Medical College, Kurnool.

4. Assistant Professor, Department of ENT, Kurnool Medical College, Kurnool.
5. Assistant Professor, Department of ENT, Kurnool Medical College, Kurnool.

\section{NAME ADDRESS EMAIL ID OF THE} CORRESPONDING AUTHOR:

Dr. S. Muneeruddin Ahmed, Professor and HOD, Department of ENT, 44/118, Prakash Nagar, Kurnool - 518004, A.P.

Email: ahmedmunirent@gmail.com

Date of Submission: 17/07/2014. Date of Peer Review: 18/07/2014. Date of Acceptance: 21/07/2014. Date of Publishing: 23/07/2014. 